\title{
Fibroblast Growth Factor Receptor 2: Expression, Roles, and Potential As a Novel Molecular Target for Colorectal Cancer
}

\author{
Yoko Matsuda, ${ }^{1}$ Junji Ueda, ${ }^{1,2}$ and Toshiyuki Ishiwata ${ }^{1}$ \\ ${ }^{1}$ Departments of Pathology and Integrative Oncological Pathology, Nippon Medical School, 1-1-5 Sendagi, Bunkyo-ku, \\ Tokyo 113-8602, Japan \\ ${ }^{2}$ Surgery for Organ and Biological Regulation, Graduate School of Medicine, Nippon Medical School, Tokyo 113-8602, Japan
}

Correspondence should be addressed to Toshiyuki Ishiwata, ishiwata@nms.ac.jp

Received 7 December 2011; Accepted 28 March 2012

Academic Editor: Gary Tse

Copyright ( 2012 Yoko Matsuda et al. This is an open access article distributed under the Creative Commons Attribution License, which permits unrestricted use, distribution, and reproduction in any medium, provided the original work is properly cited.

\begin{abstract}
The fibroblast growth factor receptor (FGFR) family consists of four members, named FGFR1, 2, 3, and 4 . All 4 FGFRs and their ligands, fibroblast growth factors (FGFs), are expressed in colorectal cancer (CRC). Recent studies have shown that FGFR2 plays important roles in cancer progression; therefore, it is of great interest as a novel target for cancers. Expression of FGFR2 regulates migration, invasion, and growth in CRC. Expression of the FGFR2 isoform FGFR2 IIIb was associated with well-differentiated histological types, and its specific ligand, FGF7, enhanced angiogenesis and adhesion to type-IV collagen via FGFR2 IIIb in CRC. FGFR2 IIIc is detected in CRC, but its roles have not been well elucidated. Interactions between FGFR2 IIIb and IIIc and FGFs may play important roles in CRC via autocrine and/or paracrine signaling. Several kinds of molecular-targeting agents against FGFR2 have been developed; however, it is not clear how a cancer treatment can most effectively inhibit FGFR2 IIIb or FGFR2 IIIc, or both isoforms. The aim of this paper is to summarize the roles of FGFR2 and its isoforms in CRC and clarify whether they are potent therapeutic targets for CRC.
\end{abstract}

\section{Introduction}

Colorectal cancer (CRC) is the second leading cause of death from cancer in the USA [1] and one of the most serious causes of death from cancer in the world. The prognosis remains poor, especially for patients with advanced or recurrent states of CRC. To improve the survival rates of patients with advanced stages, several types of anticancer, moleculartargeted agents have been developed and are currently undergoing clinical trials [2].

The fibroblast growth factors (FGFs) are heparin-binding growth factors and are classified as FGF-1 to FGF-23 [3-5]. Human FGFs, which comprise $\sim 150-300$ amino acids, have a conserved $\sim 120$ amino acid residue core, containing $\sim 30$ $60 \%$ amino acid identity [4]. Patients with CRC have been reported to overexpress FGF-1 (acidic FGF), FGF-2 (basic FGF), FGF-3, FGF-7 (keratinocyte growth factor/KGF), FGF9, FGF-10, FGF-18, FGF-19, and FGF-20. FGFs exert their biological activities by binding to high-affinity tyrosine kinase FGF receptors (FGFRs) on the surface of cells and low-affinity heparan sulfate proteoglycans that enhance ligand presentation [4]. FGFRs consist of four members, named FGFR1, 2, 3, and 4, that are encoded by distinct genes. FGFRs are single transmembrane receptors, containing extracellular, transmembrane, and intracellular domains. The extracellular domain of FGFRs is usually composed of 3 immunoglobulinlike domains (I-III). Alternative splicing of the C-terminal half of the third Ig-like domain generates the IIIb and IIIc isoforms in FGFRs1-3, but FGFR4 does not possess such alternative exons. There are reports of FGFRs $1-4$ being expressed in CRC [6-11]. The FGF/FGFR family plays important roles in development and differentiation in normal human tissues, and the family is deeply involved in carcinogenesis and cancer progression in an autocrine or paracrine manner.

Recent studies have shown that gene amplification, abnormal activation, or single nucleotide polymorphisms (SNPs) of FGFR2 play important roles in cancer progression [12-16]; therefore, FGFR2 has been recognized as a novel therapeutic target for cancers [17]. The aim of this paper is to summarize the roles of FGFR2 and its isoforms in CRC 


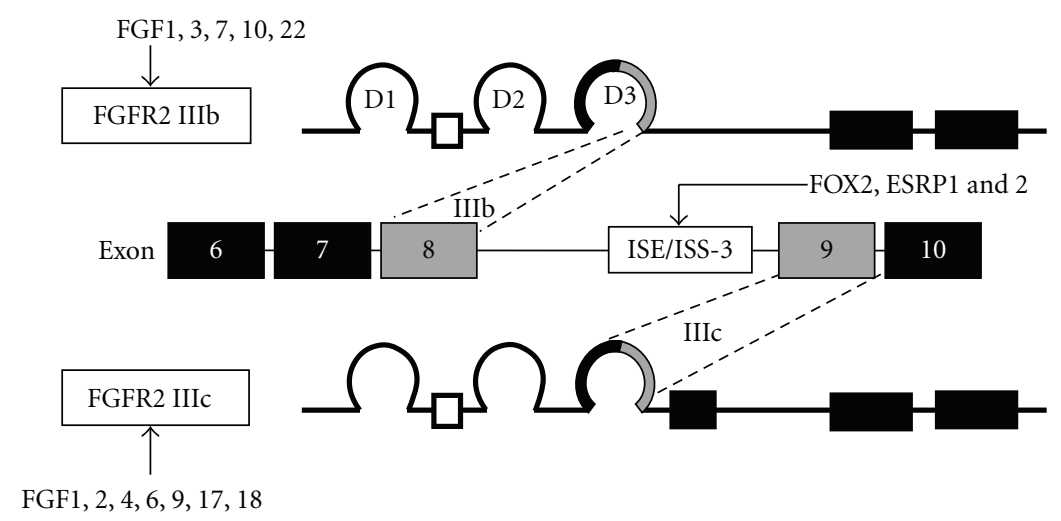

FIGURE 1: Alternative splicing of FGFR2 and specific ligands of each variant. The two FGFR2 isoforms are generated by alternative splicing of exons 8 and 9. This alternative splicing determines the specific ligands for each FGFR2 isoform [19]. FOX-2 or ESRP1 and 2 bind to the ISE/ISS-3 element to enhance splicing of exon 8 and silencing of exon 9.

and clarify whether they are potential therapeutic targets for CRC.

\section{Structure of FGFR2 and Its Isoforms}

An important feature and mode of regulation of FGFR2 functions is that structural variants of FGFR2 are generated by numerous alternative gene splicing events. These alternative splicing events of FGFR2 pre-mRNA generate mature mRNA, which encodes proteins altered in both the extracellular and intracellular regions. To date, more than 20 alternative splicing variants of FGFR2 have been identified [18].

The major splicing event occurs in the carboxyl terminal half of the third Ig-like domain (D3). The two types of FGFR2 isoforms are generated by alternative splicing of exons 8 and 9. When the C-terminal half of D3 is encoded by exon 8 , the FGFR2 IIIb isoform is generated; while the FGFR2 IIIc isoform is generated when the C-terminal half of D3 is encoded by exon 9 (Figure 1) [19]. The Intronic Splicing Enhancer/Intronic Splicing Silencer-3 (ISE/ISS-3), which is located in intron 8 downstream of a UGCAUG motif of FGFR2, regulates the FGFR2 splicing [20] via binding of FOX-2 [21] or Epithelial Splicing Regulatory Protein (ESRP) 1 and 2 [22]. The ISE/ISS-3 functions specifically in epithelial cell types to enhance splicing of the upstream exon 8 and silencing of the downstream exon 9 [23]. Tissue-specific inclusion of either exon 8 or 9 generates either the epithelial cell-specific IIIb or mesenchymal cell-specific IIIc isoforms [24]. Subsequently, this alternative splicing determines the specific ligands for each FGFR2 isoform. FGF-1, 3, 7, 10, and 22 are reported to bind to FGFR2 IIIb; while FGF-1, 2, 4, $6,9,17$, and 18 bind to FGFR2 IIIc with high affinity [19, $25,26]$. FGF-FGFR binding activates intracellular signaling cascades. Mitogenic signaling is mediated through tyrosine phosphorylation of key substrates, including activation of the mitogen-activated protein kinases, such as ERK-1 and ERK-2 via the ras pathway $[27,28]$.

The other major splicing of FGFR2 occurs in the sequence encoding the intracytoplasmic carboxyl terminus of FGFR2 IIIb. Three splice variants of FGFR2 IIIb have been identified. The variants are named C1, C2, and C3, and each has a different carboxyl-terminal sequence [29]. The C2-type carboxyl terminus is 34 amino acids shorter than the C1-type carboxyl terminus, and the C3-type carboxyl terminus is 19 amino acids shorter than the C2-type carboxyl terminus. These sequence differences result in distinct retention of tyrosine residues that may serve as sites for receptor autophosphorylation and docking sites for the cytoplasmic signaling proteins of FGFR2 IIIb. The FGFR2 IIIb-C3 isoform lacks the putative phospholipase $\mathrm{C} \gamma$ (PLC $\gamma$ ) binding site.

\section{Expression and Roles of FGFR2 in Various Cancers}

There are many published reports concerning the expression of FGFR2 in various cancers. Previous reports have shown overexpression of the C3 isoform in gastric cancer cell lines [29] and the C2 and C3 isoforms in breast cancer cell lines $[30,31]$, suggesting that aberrant expression of C2 or C3 splicing variants may contribute to cancer development.

Anomalous FGF signaling is associated with cancer development and progression. Gene amplification or missense mutations of FGFR2 occur in gastric, lung, breast, ovarian, and endometrial cancers and melanomas $[8,15,18$, 32-35]. SNPs of intron 2 in FGFR2 are associated with an increased risk of breast and endometrial cancers [12-14]. Furthermore, activating mutations of FGFR2 have been identified in approximately $10 \%$ of endometrial cancers, and inhibition of activated mutations of FGFR2 induced apoptosis and growth inhibition of endometrial carcinoma cells [15, 16]. In contrast, loss-of-function mutations of FGFR2 have been reported in melanomas [35]. These findings suggest that FGFR2 can play a context-dependent, opposing roles in various cancers.

A class switch from FGFR2 IIIb to FGFR2 IIIc is related to the progression of prostate cancers [36]. Furthermore, FGFR2 IIIc expression in prostate and bladder cancer cells induced epithelial-mesenchymal transition (EMT) and a switch in splicing, which may play crucial roles in cancer 

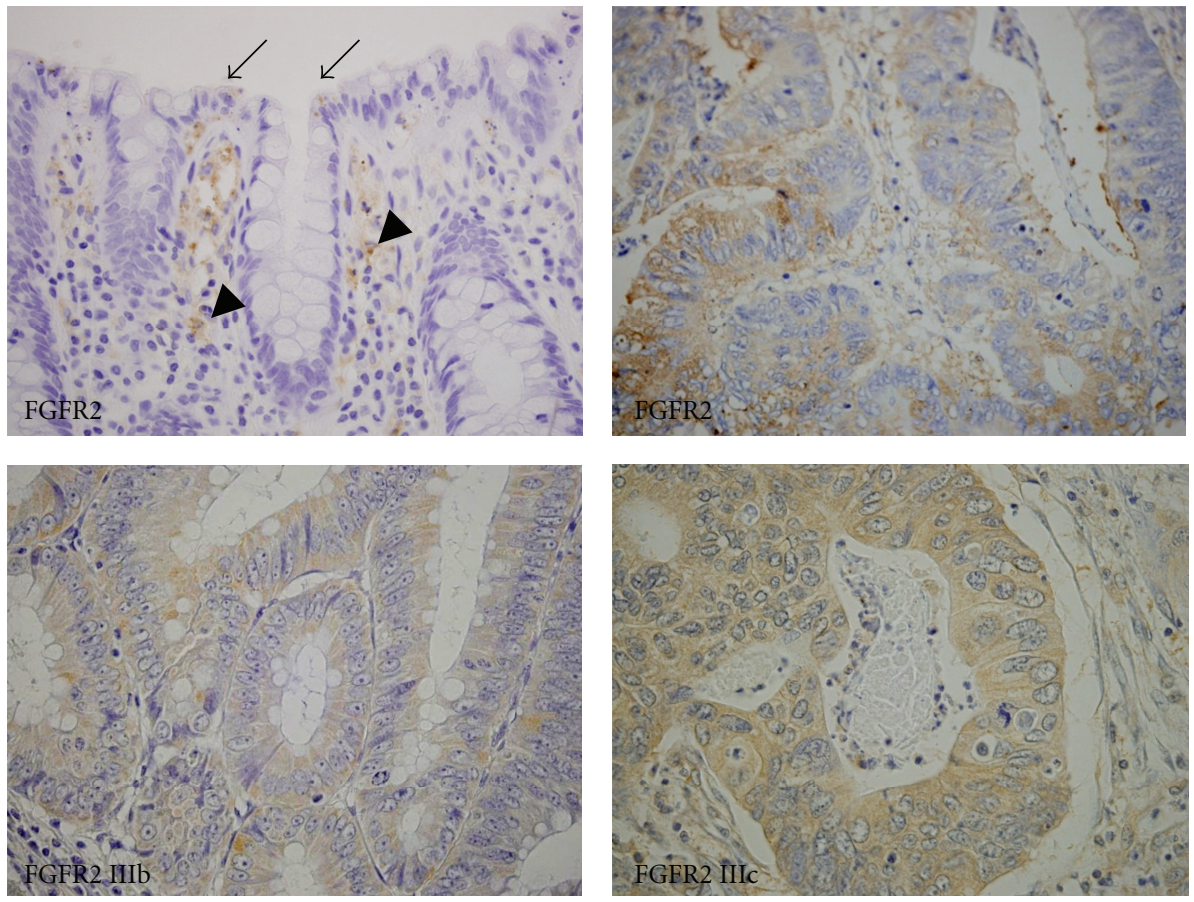

FIGURE 2: Immunohistochemical images of FGFR2 and its isoforms FGFR2 IIIb and FGFR2 IIIc in CRC. FGFR2 was expressed in the surface area of crypts in a nontumorous lesion obtained from CRC tissue (arrows). FGFR2 was also expressed in fibroblasts, inflammatory cells, and vascular endothelium (arrowheads). CRC cells exhibited marked expression of FGFR2 in the cell membrane and cytoplasm. Isoforms of FGFR2, FGFR2 IIIb, and FGFR2 IIIc were expressed in CRC cells. Original magnification: upper left panel, $\times 200$; other panels, $\times 600$.

metastasis [37-39]. We previously reported that the expression levels of FGFR2 positively correlated with the presence of precancerous lesions in the uterine cervix, termed cervical intraepithelial neoplasia (CIN). Furthermore, stable transfection of FGFR2 IIIc in cervical cancer cell lines induced cancer cell growth [40]. Therefore, FGFR2 IIIc correlates with the carcinogenesis and aggressive growth of cervical cancer.

In contrast, the roles of the FGFR2 IIIb isoform have been controversial. Overexpression of the FGFR2 IIIb isoform, also known as keratinocyte growth factor receptor, has been reported in various cancers, including breast, endometrial, cervical, lung, esophageal, gastric, pancreatic, and CRC [10, 41-51]. We reported that expression of FGFR2 IIIb and one of its major ligands, FGF7, correlated with venous invasion, vascular endothelial growth factor A (VEGF-A) expression, and a poor prognosis and may promote venous invasion and tumor angiogenesis in pancreatic cancers [49]. Pancreatic cancer patients with high FGFR2 expression had a shorter survival time compared to those with low FGFR2 expression. Another ligand for FGFR2 IIIb, FGF10, induced pancreatic cancer cell migration and invasion via FGFR2 IIIb [52]. In contrast, decreased expression of FGFR2 IIIb in gastric cancer cells was associated with the proliferation and invasion of gastric cancer cells and a poor prognosis for the patient [47]. In esophageal cancers, FGFR2 IIIb expression correlated with a well-differentiated cell type, and FGF7 induced cell proliferation in FGFR2 IIIb positive cancer cells [10]. The different roles of FGFR2 IIIb in various cancers have not been well characterized; however, differences may be due to the affinity of different ligands for FGFR2 IIIb or effects from other FGFRs.

\section{Expression and Roles of FGFR2 in CRC}

Immunohistochemical analysis has demonstrated that FGFR2 is expressed in the differentiated cells located on the upper portion of the intestinal crypt in normal colorectal epithelium (Figure 2, arrows) [53] and is related to the proliferation and differentiation of cells $[10,54]$. In patients with CRC, expression of FGFR2 protein was observed in the cell membrane and cytoplasm of cancer cells (Figure 2). The mRNA of several FGFs, including FGF2, 8, 9, and 18, was expressed in CRC cell lines and was detected by RT-PCR analysis (Table 1) [49]; therefore, FGFs/FGFRs may regulate CRC cell growth in an autocrine manner. Recently, we reported that the invasive front of CRC cells exhibited stronger FGFR2 expression than the surface or central area of the cancerous cells [53]. Decreased expression of FGFR2 in CRC cells was associated with inhibited cell migration, invasion, and tumor growth in vitro and in vivo [53]. Thus, FGFR2 plays important roles in CRC progression in correlation with tumor cell migration, invasion, and growth (Table 2).

Overexpression of the FGFR2 isoform FGFR2 IIIb in CRC was associated with a well-differentiated histological type $[10,55]$. As determined by in situ hybridization, both FGFR2 IIIb and FGF7 mRNA are expressed in CRC cells, and 
TABLE 1: Expression of FGFs in CRC cell lines.

\begin{tabular}{|c|c|c|c|c|c|c|c|c|}
\hline & FGF1 & FGF2 & FGF4 & FGF6 & FGF8 & FGF9 & FGF17 & FGF18 \\
\hline DLD-1 & - & + & - & - & + & - & - & + \\
\hline HCT-15 & - & + & - & - & + & + & - & + \\
\hline SW480 & - & - & - & - & + & + & - & + \\
\hline LoVo & - & + & - & - & + & + & - & - \\
\hline Colo201 & - & - & - & - & + & + & - & + \\
\hline
\end{tabular}

TABLE 2: Roles of FGFR2 and its splicing variants in colorectal cancers.

\begin{tabular}{llcc}
\hline & & Reported data & Roles \\
\hline FGFR2 & $\begin{array}{c}\text { normal } \\
\text { cancer }\end{array}$ & $\begin{array}{c}\text { located in the upper portion of the intestinal crypt [50, 54] } \\
\text { increased expression in the invasive front of cancer cells } \\
\text { shRNA-targeting FGFR2 suppressed cell growth [53] }\end{array}$ & $\begin{array}{c}\text { differentiation, growth } \\
\text { invasion, growth }\end{array}$ \\
\hline FGFR2 IIIb & $\begin{array}{l}\text { normal } \\
\text { cancer }\end{array}$ & located in the upper portion of the intestinal crypt [50] & differentiation, growth \\
& cancer & Well-differentiated types [50] & differentiation \\
& cancer & FGF7 enhanced the adhesion to type-IV collagen [57] & angiogenesis \\
FGFR2 IIIc & cancer & FGF10 increased the growth rate [51] & growth \\
\hline
\end{tabular}

FGF7 mRNA was recognized in neuroendocrine cells lying close to CRC cells [50]. FGF7 induced an increase of VEGFA; thus, FGF-7 and its receptor FGFR2 IIIb may be involved in tumor angiogenesis in CRC [56]. Furthermore, FGF7 enhances adhesion to type-IV collagen, one of the main components of the vascular basement membrane, via downregulation of Integrin $\alpha 2$ and activation of ERK1/2 and focal adhesion kinase (FAK) signaling pathways [57]. Coexpression of FGFR2 IIIb and FGF10 has been reported in CRC cells, and FGF10 increased the growth rate of FGFR2 IIIbpositive CRC cell lines [51]. These findings suggest interactions of FGFR2 IIIb, FGF7, and FGF10 may play important roles in CRC proliferation and tumor angiogenesis via autocrine and/or paracrine manners (Table 2).

Immunohistochemical analysis, quantitative RT-PCR, and fluorescent staining determined that both FGFR2 IIIb and FGFR2 IIIc are expressed in CRC tissues and cell lines (Figures 2, 3, and 4) [49, 58]. However, the roles of the FGFR2 IIIc isoform in CRC are not clear and require further examination.

\section{Molecular-Targeted Therapy of the FGFR2 Signaling Pathway}

Several kinds of molecular-targeted therapies to the FGFR2 signaling pathway have been reported in various cancers. In endometrial cancer cells harboring activated mutations of FGFR2, knockdown of FGFR2 using short hairpin (sh) RNA or treatment with a pan-FGFR inhibitor, PD173074, caused cell cycle arrest and cell death [15]. A small molecule,

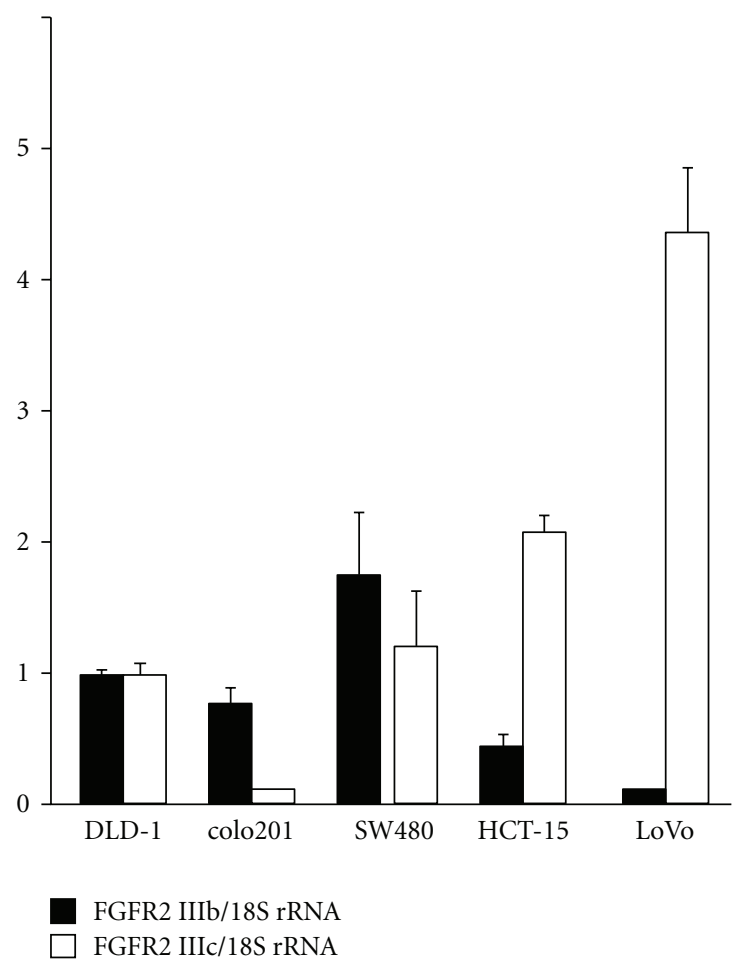

FIGURE 3: Expression levels of FGFR2 IIIb and IIIc in CRC cell lines. All 5 CRC cell lines expressed FGFR2 IIIb and IIIc mRNAs at various levels. Quantitative RT-PCR results were expressed as the ratio of target to $18 \mathrm{~S}$ rRNA, with the latter serving as an internal standard. Gene expression levels were measured in triplicate. 

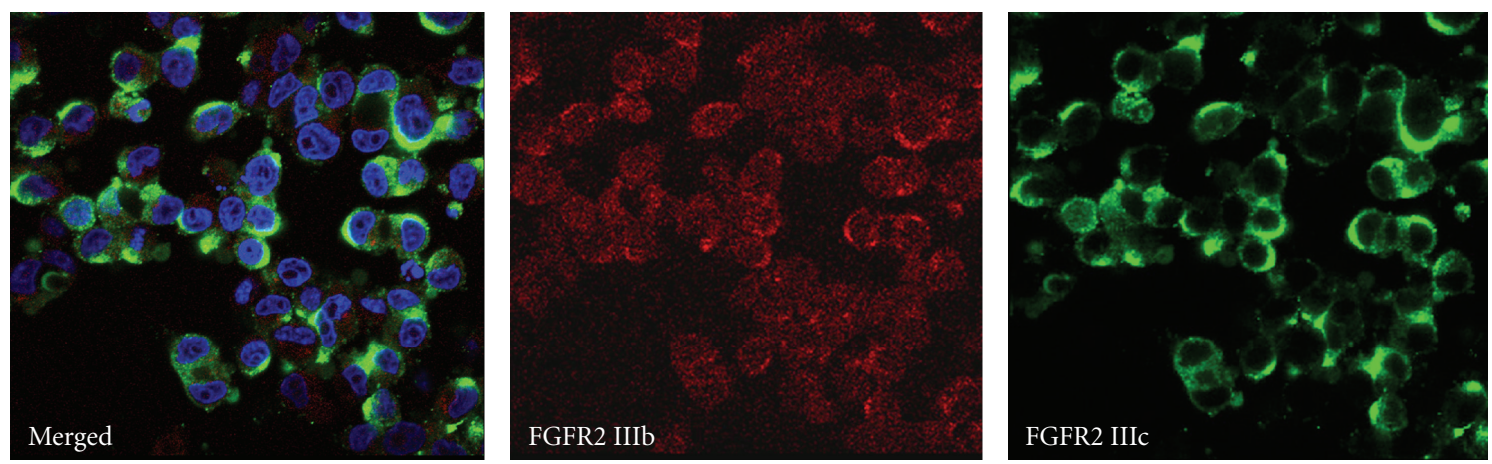

FIgURE 4: Immunocytochemical analysis of FGFR2 IIIb and IIIc in CRC cell lines. A human CRC cell line, LoVo, exhibited both FGFR2 IIIb and FGFR2 IIIc isoforms in the cell membrane and cytoplasm. The expression level of FGFR2 IIIb was weak, but IIIc was strong, which is consistent with their mRNA levels. Original magnification, $\times 1000$; red, FGFR2 IIIb; green, FGFR2 IIIc; blue, DAPI.

Ki23057, which inhibits autophosphorylation of FGFR2 IIIb decreased the growth of biliary tract cancer cells [59], gastric scirrhous carcinoma cells [60,61], and CRC cells [62] in vitro and in vivo. A recent study demonstrated that monoclonal antibodies to FGFR2 IIIb and FGFR2 IIIc isoforms successfully inhibited the growth of gastric tumor xenografts [63]. A mutation in the soluble ectodomain of FGFR2 IIIc, S252W, suppressed cell growth, angiogenesis, and metastasis of human breast cancer and prostate cancer cell lines in vitro and in vivo [64]. We recently reported that shRNA-targeting FGFR2 in CRC cell lines suppressed cancer cell growth, migration, and invasion [53]; however, no other reports have shown the usefulness of FGFR2-targeting therapy in CRC. The best way for cancer treatment to inhibit FGFR2 IIIb or FGFR2 IIIc, or both isoforms, has not been determined. It may depend on each cell line or individuals that express different endogenous FGFR2 levels, and other FGFRs and FGFs. Basic research clearly indicates the effectiveness of FGFR2 as a target for CRC therapy; therefore, clinical studies are needed to develop a novel therapeutic strategy in CRC.

\section{Other FGFRs and FGFs in CRC}

All four FGFRs are reportedly expressed in CRC [6-11]. FGFR1 and FGFR2 are expressed in both colorectal adenomas and CRC, and expression might indicate the transformation from human colon adenoma to carcinoma [6]. Overexpression of the FGFR1 gene leads to liver metastasis in CRC [9].

FGF2 and FGF20 are implicated in embryogenesis and tissue regeneration within the colon [65-67]. FGF2 is expressed in submucosal tissues to induce angiogenesis, while FGF20 is expressed in epithelial progenitor cells to induce epithelial proliferation. Aberrant splicing and activation of cryptic splice sequences in FGFR3 have been identified in patients with CRC $[8,68]$. FGFR4 and its specific ligand FGF19 are coexpressed in cancer cells and induce growth via ERK and FRS2 in CRC and hepatocellular carcinoma [69]. In human intestinal epithelial cell lines and CRC cell lines, a soluble splice variant of FGFR4 has been reported [70], but its roles have not been determined.

\section{Molecular-Targeted Therapy to Other FGF/FGFR Signaling Pathways}

Protein kinases all have a catalytic domain with a conserved amino acid sequence and similar three-dimensional structures [71]. Protein kinases are now the most popular class of drug target after $\mathrm{G}$ protein-coupled receptors [72]. Smallmolecule compounds with multiple targets have recently been developed to overcome the recurrence of drug-resistant tumors [73]. There are several inhibitors of FGFR1 tyrosine kinase activity, including PD173074, SU5402, and AZD2171. AZD2171 inhibits VEGFR2, PDGFR $\beta$, and FGFR1 tyrosine kinase activity, while PD173074 and SU5402 target a relatively narrow range of tyrosine kinases. In a mousexenografted CRC tumor, combined therapy with a recombinant FGFR1 protein vaccine and low-dose gemcitabine inhibited tumor growth and antiangiogenesis was present [74]. TSU68, a multiple-receptor tyrosine kinase inhibitor that targets FGFRs, vascular endothelial growth factor receptor-2 (VEGFR2), and a platelet-derived growth factor receptor (PDGFR) inhibited CRC cell growth via normalization of tumor vessels [75]. Brivanib Alaninate is an orally available, selective tyrosine kinase inhibitor that targets both VEGFR-2 and FGFR1 [76]. ENMD-2076, a small-molecule kinase inhibitor with activity against the Aurora kinases A and $\mathrm{B}$ and several other tyrosine kinases, including VEGFR2, cKit, and FGFR1, inhibited tumor growth in murine xenograft models of human CRC [77].

An anti-FGFR3 monoclonal antibody that inhibited not only wild-type FGFR3, but also various mutants of the receptor, exerted potent antitumor activity against bladder carcinoma and multiple myeloma xenografts in mice [78]. The human single-chain variable fragment $(\mathrm{Fv})$ directed against FGFR3 blocked the proliferation of bladder carcinoma cells in vitro and in vivo [79]. Currently, most small molecule compounds inhibit multiple tyrosine kinases and may affect not only cancer cells but also normal cells. To develop more selective tyrosine kinase inhibitors, monoclonal antibodies, vaccines, and emerging technologies, such as RNA aptamers or siRNA, will be needed to treat advanced CRC. 


\section{Conclusion}

FGFR2 and its isoform are highly expressed in CRC and correlate with CRC growth, invasion, and tumor angiogenesis. The effectiveness of FGFR2-targeting therapy for CRC has been clearly demonstrated in vitro and in vivo in basic research studies, more so than for other FGFs or FGFRs. Thus, anti-FGFR2 therapies, including selective targeted therapy to the isoforms, may be novel treatments for advanced CRC patients.

\section{Acknowledgments}

The authors thank Dr. Zenya Naito (Departments of Pathology and Integrative Oncological Pathology, Nippon Medical School) and Dr. Yoshiharu Ohaki (Department of Pathology, Chiba-Hokusoh Hospital, Nippon Medical School) for helpful discussion.

\section{References}

[1] R. Siegel et al., "Cancer statistics, 2011: the impact of eliminating socioeconomic and racial disparities on premature cancer deaths," CA-Cancer Journal for Clinicians, vol. 61, no. 4, pp. 212-236, 2011.

[2] I. Chau and D. Cunningham, "Treatment in advanced colorectal cancer: what, when and how?" British Journal of Cancer, vol. 100, no. 11, pp. 1704-1719, 2009.

[3] N. Itoh, "The Fgf families in humans, mice, and zebrafish: their evolutional processes and roles in development, metabolism, and disease," Biological and Pharmaceutical Bulletin, vol. 30, no. 10, pp. 1819-1825, 2007.

[4] N. Itoh and D. M. Ornitz, "Evolution of the Fgf and Fgfr gene families," Trends in Genetics, vol. 20, no. 11, pp. 563-569, 2004.

[5] M. Katoh and M. Katoh, "FGF signaling network in the gastrointestinal tract (Review)," International Journal of Oncology, vol. 29, no. 1, pp. 163-168, 2006.

[6] G. C. Jayson et al., "Coordinated modulation of the fibroblast growth factor dual receptor mechanism during transformation from human colon adenoma to carcinoma," International Journal of Cancer, vol. 82, no. 2, pp. 298-304, 1999.

[7] M. Spinola, V. P. Leoni, J. I. Tanuma et al., "FGFR4 Gly388Arg polymorphism and prognosis of breast and colorectal cancer," Oncology Reports, vol. 14, no. 2, pp. 415-419, 2005.

[8] J. H. Jang, K. H. Shin, and J. G. Park, "Mutations in fibroblast growth factor receptor 2 and fibroblast growth factor receptor 3 genes associated with human gastric and colorectal cancers," Cancer Research, vol. 61, no. 9, pp. 3541-3543, 2001.

[9] T. Sato, T. Oshima, K. Yoshihara et al., "Overexpression of the fibroblast growth factor receptor-1 gene correlates with liver metastasis in colorectal cancer," Oncology Reports, vol. 21, no. 1, pp. 211-216, 2009.

[10] M. Yoshino, T. Ishiwata, M. Watanabe et al., "Keratinocyte growth factor receptor expression in normal colorectal epithelial cells and differentiated type of colorectal cancer," Oncology Reports, vol. 13, no. 2, pp. 247-252, 2005.

[11] G. Sonvilla, S. Allerstorfer, C. Heinzle et al., "Fibroblast growth factor receptor 3-IIIc mediates colorectal cancer growth and migration," British Journal of Cancer, vol. 102, no. 7, pp. 1145$1156,2010$.

[12] D. F. Easton et al., "Genome-wide association study identifies novel breast cancer susceptibility loci," Nature, vol. 447, no. 7148, pp. 1087-1093, 2007.
[13] D. J. Hunter, P. Kraft, K. B. Jacobs et al., "A genome-wide association study identifies alleles in FGFR2 associated with risk of sporadic postmenopausal breast cancer," Nature Genetics, vol. 39, no. 7, pp. 870-874, 2007.

[14] M. McGrath, I. M. Lee, J. Buring, D. J. Hunter, and I. De Vivo, "Novel breast cancer risk alleles and endometrial cancer risk," International Journal of Cancer, vol. 123, no. 12, pp. 29612964, 2008.

[15] S. A. Byron, M. G. Gartside, C. L. Wellens et al., "Inhibition of activated fibroblast growth factor receptor 2 in endometrial cancer cells induces cell death despite PTEN abrogation," Cancer Research, vol. 68, no. 17, pp. 6902-6907, 2008.

[16] S. A. Byron and P. M. Pollock, "FGFR2 as a molecular target in endometrial cancer," Future Oncology, vol. 5, no. 1, pp. 27-32, 2009.

[17] Y. Katoh and M. Katoh, "FGFR2-related pathogenesis and FGFR2-targeted therapeutics (review)," International Journal of Molecular Medicine, vol. 23, no. 3, pp. 307-311, 2009.

[18] M. Katoh, "Cancer genomics and genetics of FGFR2 (Review)," International Journal of Oncology, vol. 33, no. 2, pp. 233-237, 2008.

[19] V. P. Eswarakumar, I. Lax, and J. Schlessinger, "Cellular signaling by fibroblast growth factor receptors," Cytokine and Growth Factor Reviews, vol. 16, no. 2, pp. 139-149, 2005.

[20] R. H. Hovhannisyan, C. C. Warzecha, and R. P. Carstens, "Characterization of sequences and mechanisms through which ISE/ISS-3 regulates FGFR2 splicing," Nucleic Acids Research, vol. 34, no. 1, pp. 373-385, 2006.

[21] A. P. Baraniak, J. R. Chen, and M. A. Garcia-Blanco, "Fox2 mediates epithelial cell-specific fibroblast growth factor receptor 2 exon choice," Molecular and Cellular Biology, vol. 26, no. 4, pp. 1209-1222, 2006.

[22] C. C. Warzecha, T. K. Sato, B. Nabet, J. B. Hogenesch, and R. P. Carstens, "ESRP1 and ESRP2 are epithelial cell-type-specific regulators of FGFR2 splicing," Molecular Cell, vol. 33, no. 5, pp. 591-601, 2009.

[23] R. H. Hovhannisyan and R. P. Carstens, "A novel intronic cis element, ISE/ISS-3, regulates rat fibroblast growth factor receptor 2 splicing through activation of an upstream exon and repression of a downstream exon containing a noncanonical branch point sequence," Molecular and Cellular Biology, vol. 25, no. 1, pp. 250-263, 2005.

[24] A. Orr-Urtreger, M. T. Bedford, T. Burakova et al., "Developmental localization of the splicing alternatives of fibroblast growth factor receptor-2 (FGFR2)," Developmental Biology, vol. 158, no. 2, pp. 475-486, 1993.

[25] O. A. Ibrahimi, A. V. Eliseenkova, A. N. Plotnikov, K. Yu, D. M. Ornitz, and M. Mohammadi, "Structural basis for fibroblast growth factor receptor 2 activation in Apert syndrome," Proceedings of the National Academy of Sciences of the United States of America, vol. 98, no. 13, pp. 7182-7187, 2001.

[26] M. Mohammadi, S. K. Olsen, and O. A. Ibrahimi, "Structural basis for fibroblast growth factor receptor activation," Cytokine and Growth Factor Reviews, vol. 16, no. 2, pp. 107-137, 2005.

[27] R. E. Friesel and T. Maciag, "Molecular mechanisms of angiogenesis: fibroblast growth factor signal transduction," FASEB Journal, vol. 9, no. 10, pp. 919-925, 1995.

[28] H. Kouhara, Y. R. Hadari, T. Spivak-Kroizman et al., "A lipid-anchored Grb2-binding protein that links FGF-receptor activation to the Ras/MAPK signaling pathway," Cell, vol. 89, no. 5, pp. 693-702, 1997.

[29] H. Itoh, Y. Hattori, H. Sakamoto et al., "Preferential alternative splicing in cancer generates a K-sam messenger RNA with 
higher transforming activity," Cancer Research, vol. 54, no. 12, pp. 3237-3241, 1994.

[30] J. Y. Cha, Q. T. Lambert, G. W. Reuther, and C. J. Der, "Involvement of fibroblast growth factor receptor 2 isoform switching in mammary oncogenesis," Molecular Cancer Research, vol. 6, no. 3, pp. 435-445, 2008.

[31] X. Zhu, S. L. Asa, and S. Ezzat, "Histone-acetylated control of fibroblast growth factor receptor 2 intron 2 polymorphisms and isoform splicing in breast cancer," Molecular Endocrinology, vol. 23, no. 9, pp. 1397-1405, 2009.

[32] H. Davies et al., "Somatic mutations of the protein kinase gene family in human lung cancer," Cancer Research, vol. 65, no. 17, pp. 7591-7595, 2005.

[33] P. M. Pollock, M. G. Gartside, L. C. Dejeza et al., "Frequent activating FGFR2 mutations in endometrial carcinomas parallel germline mutations associated with craniosynostosis and skeletal dysplasia syndromes," Oncogene, vol. 26, no. 50, pp. 7158-7162, 2007.

[34] J. Adnane, P. Gaudray, C. A. Dionne et al., "BEK and FLG, two receptors to members of the FGF family, are amplified in subsets of human breast cancers," Oncogene, vol. 6, no. 4, pp. 659-663, 1991.

[35] M. G. Gartside et al., "Loss-of-function fibroblast growth factor receptor-2 mutations in melanoma," Molecular Cancer Research, vol. 7, no. 1, pp. 41-54, 2009.

[36] R. P. Carstens, J. V. Eaton, H. R. Krigman, P. J. Walther, and M. A. Garcia-Blanco, "Alternative splicing of fibroblast growth factor receptor 2 (FGF-R2) in human prostate cancer," Oncogene, vol. 15, no. 25, pp. 3059-3065, 1997.

[37] C. L. Chaffer, B. Dopheide, P. Savagner, E. W. Thompson, and E. D. Williams, "Aberrant fibroblast growth factor receptor signaling in bladder and other cancers," Differentiation, vol. 75, no. 9, pp. 831-842, 2007.

[38] C. L. Chaffer, J. P. Brennan, J. L. Slavin, T. Blick, E. W. Thompson, and E. D. Williams, "Mesenchymal-to-epithelial transition facilitates bladder cancer metastasis: role of fibroblast growth factor receptor-2," Cancer Research, vol. 66, no. 23, pp. 11271-11278, 2006.

[39] S. Oltean, B. S. Sorg, T. Albrecht et al., "Alternative inclusion of fibroblast growth factor receptor 2 exon IIIc in Dunning prostrate tumors reveals unexpected epithelial mesenchymal plasticity," Proceedings of the National Academy of Sciences of the United States of America, vol. 103, no. 38, pp. 14116-14121, 2006.

[40] T. Kawase, K. Matsuo, T. Suzuki et al., "FGFR2 intronic polymorphisms interact with reproductive risk factors of breast cancer: results of a case control study in Japan," International Journal of Cancer, vol. 125, no. 8, pp. 1946-1952, 2009.

[41] Y. A. Luqmani, M. Graham, and R. C. Coombes, "Expression of basic fibroblast growth factor, FGFR1 and FGFR2 in normal and malignant human breast, and comparison with other normal tissues," British Journal of Cancer, vol. 66, no. 2, pp. 273-280, 1992.

[42] A. Ishikawa, M. Kudo, N. Nakazawa et al., "Expression of keratinocyte growth factor and its receptor in human endometrial cancer in cooperation with steroid hormones," International Journal of Oncology, vol. 32, no. 3, pp. 565-574, 2008.

[43] G. Kurban, T. Ishiwata, M. Kudo, M. Yokoyama, Y. Sugisaki, and Z. Naito, "Expression of keratinocyte growth factor receptor (KGFR/FGFR2 IIIb) in human uterine cervical cancer," Oncology Reports, vol. 11, no. 5, pp. 987-991, 2004.

[44] R. Kawase, T. Ishiwata, Y. Matsuda et al., "Expression of fibroblast growth factor receptor 2 IIIc in human uterine cervical intraepithelial neoplasia and cervical cancer," International Journal of Oncology, vol. 36, no. 2, pp. 331-340, 2010.

[45] T. Yamayoshi, T. Nagayasu, K. Matsumoto, T. Abo, Y. Hishikawa, and T. Koji, "Expression of keratinocyte growth factor/fibroblast growth factor-7 and its receptor in human lung cancer: correlation with tumour proliferative activity and patient prognosis," Journal of Pathology, vol. 204, no. 1, pp. 110-118, 2004.

[46] M. Yoshino, T. Ishiwata, M. Watanabe et al., "Expression and roles of keratinocyte growth factor and its receptor in esophageal cancer cells," International Journal of Oncology, vol. 31, no. 4, pp. 721-728, 2007.

[47] T. Matsunobu, T. Ishiwata, M. Yoshino et al., "Expression of keratinocyte growth factor receptor correlates with expansive growth and early stage of gastric cancer," International Journal of Oncology, vol. 28, no. 2, pp. 307-314, 2006.

[48] T. Ishiwata, H. Friess, M. W. Büchler, M. E. Lopez, and M. Korc, "Characterization of keratinocyte growth factor and receptor expression in human pancreatic cancer," American Journal of Pathology, vol. 153, no. 1, pp. 213-222, 1998.

[49] K. Cho, T. Ishiwata, E. Uchida et al., "Enhanced expression of keratinocyte growth factor and its receptor correlates with venous invasion in pancreatic cancer," American Journal of Pathology, vol. 170, no. 6, pp. 1964-1974, 2007.

[50] M. Watanabe, T. Ishiwata, K. Nishigai, Y. Moriyama, and G. Asano, "Overexpression of keratinocyte growth factor in cancer cells and enterochromaffin cells in human colorectal cancer," Pathology International, vol. 50, no. 5, pp. 363-372, 2000.

[51] A. Matsuike, T. Ishiwata, M. Watanabe, and G. Asano, "Expression of fibroblast growth factor (FGF)-10 in human colorectal adenocarcinoma cells," Journal of Nippon Medical School, vol. 68, no. 5, pp. 397-404, 2001.

[52] S. Nomura, H. Yoshitomi, S. Takano et al., "FGF10/FGFR2 signal induces cell migration and invasion in pancreatic cancer," British Journal of Cancer, vol. 99, no. 2, pp. 305-313, 2008.

[53] Y. Matsuda, T. Ishiwata, K. Yamahatsu et al., "Overexpressed fibroblast growth factor receptor 2 in the invasive front of colorectal cancer: a potential therapeutic target in colorectal cancer," Cancer Letters, vol. 309, no. 2, pp. 209-219, 2011.

[54] V. Visco, F. Belleudi, C. Marchese et al., "Differential response to keratinocyte growth factor receptor and epidermal growth factor receptor ligands of proliferating and differentiating intestinal epithelial cells," Journal of Cellular Physiology, vol. 200, no. 1, pp. 31-44, 2004.

[55] J. M. Otte et al., "Expression of keratinocyte growth factor and its receptor in colorectal cancer," European Journal of Clinical Investigation, vol. 30, no. 3, pp. 222-229, 2000.

[56] K. Narita, T. Fujii, T. Ishiwata et al., "Keratinocyte growth factor induces vascular endothelial growth factor - A expression in colorectal cancer cells," International Journal of Oncology, vol. 34, no. 2, pp. 355-360, 2009.

[57] M. Kudo, T. Ishiwata, N. Nakazawa et al., "Keratinocyte growth factor-transfection-stimulated adhesion of colorectal cancer cells to extracellular matrices," Experimental and Molecular Pathology, vol. 83, no. 3, pp. 443-452, 2007.

[58] T. Ishiwata et al., "Enhanced expression of fibroblast growth factor receptor 2 IIIc promotes human pancreatic cancer cell proliferation," American Journal of Pathology, vol. 180, no. 5, pp. 1928-1941, 2012.

[59] R. Amano, N. Yamada, Y. Doi et al., "Significance of keratinocyte growth factor receptor in the proliferation of biliary 
tract cancer," Anticancer Research, vol. 30, no. 10, pp. 41154121, 2010.

[60] K. Nakamura, M. Yashiro, T. Matsuoka et al., "A novel molecular targeting compound as K-samII/FGF-R2 phosphorylation inhibitor, Ki23057, for scirrhous gastric cancer," Gastroenterology, vol. 131, no. 5, pp. 1530-1541, 2006.

[61] M. Yashiro, O. Shinto, K. Nakamura et al., "Synergistic antitumor effects of FGFR2 inhibitor with 5-fluorouracil on scirrhous gastric carcinoma," International Journal of Cancer, vol. 126, no. 4, pp. 1004-1016, 2010.

[62] K. Sakurai, N. Yamada, M. Yashiro et al., "A novel angiogenesis inhibitor, Ki23057, is useful for preventing the progression of colon cancer and the spreading of cancer cells to the liver," European Journal of Cancer, vol. 43, no. 17, pp. 2612-2620, 2007.

[63] W. M. Zhao, L. Wang, H. Park et al., "Monoclonal antibodies to fibroblast growth factor receptor 2 effectively inhibit growth of gastric tumor xenografts," Clinical Cancer Research, vol. 16, no. 23 , pp. 5750-5758, 2010.

[64] J. Wang et al., "Antitumor activity of a recombinant soluble ectodomain of mutant human fibroblast growth factor receptor-2 IIIc," Molecular Cancer Therapeutics, vol. 10, no. 9, pp. 1656-1666, 2011.

[65] A. M. Gonzalez, D. J. Hill, A. Logan, P. A. Maher, and A. Baird, "Distribution of fibroblast growth factor (FGF)-2 and FGF receptor-1 messenger RNA expression and protein presence in the mid-trimester human fetus," Pediatric Research, vol. 39, no. 3, pp. 375-385, 1996.

[66] H. Kirikoshi, N. Sagara, T. Saitoh et al., "Molecular cloning and characterization of human FGF-20 on chromosome 8p21.3-p22," Biochemical and Biophysical Research Communications, vol. 274, no. 2, pp. 337-343, 2000.

[67] M. Jeffers, W. F. McDonald, R. A. Chillakuru et al., "A novel human fibroblast growth factor treats experimental intestinal inflammation," Gastroenterology, vol. 123, no. 4, pp. 11511162, 2002.

[68] J. H. Jang, K. H. Shin, Y. J. Park, R. J. Lee, W. L. McKeehan, and J. G. Park, "Novel transcripts of fibroblast growth factor receptor 3 reveal aberrant splicing and activation of cryptic splice sequences in colorectal cancer," Cancer Research, vol. 60, no. 15, pp. 4049-4052, 2000.

[69] L. R. Desnoyers, R. Pai, R. E. Ferrando et al., "Targeting FGF19 inhibits tumor growth in colon cancer xenograft and FGF19 transgenic hepatocellular carcinoma models," Oncogene, vol. 27, no. 1, pp. 85-97, 2008.

[70] S. Takaishi, M. Sawada, Y. Morita, H. Seno, H. Fukuzawa, and T. Chiba, "Identification of a novel alternative splicing of human FGF receptor 4: soluble-form splice variant expressed in human gastrointestinal epithelial cells," Biochemical and Biophysical Research Communications, vol. 267, no. 2, pp. 658662,2000 .

[71] M. E. M. Noble, J. A. Endicott, and L. N. Johnson, "Protein kinase inhibitors: insights into drug design from structure," Science, vol. 303, no. 5665, pp. 1800-1805, 2004.

[72] D. S. Krause and R. A. Van Etten, "Tyrosine kinases as targets for cancer therapy," New England Journal of Medicine, vol. 353, no. 2, pp. 172-187, 2005.

[73] M. Katoh and M. Katoh, "Bioinformatics for cancer management in the post-genome era," Technology in Cancer Research and Treatment, vol. 5, no. 2, pp. 169-175, 2006.

[74] S. J. Zheng, S. P. Zheng, F. Y. Huang, C. L. Jiao, and R. L. $\mathrm{Wu}$, "Synergistic anti-tumor effect of recombinant chicken fibroblast growth receptor-1-mediated anti-angiogenesis and low-dose gemcitabine in a mouse colon adenocarcinoma model," World Journal of Gastroenterology, vol. 13, no. 17, pp. 2484-2489, 2007.

[75] M. Ohta, T. Kawabata, M. Yamamoto et al., "TSU68, an antiangiogenic receptor tyrosine kinase inhibitor, induces tumor vascular normalization in a human cancer xenograft nude mouse model," Surgery Today, vol. 39, no. 12, pp. 1046-1053, 2009.

[76] M. Ayers, J. Fargnoli, A. Lewin, Q. Wu, and J. Suso Platero, "Discovery and validation of biomarkers that respond to treatment with brivanib alaninate, a small-molecule VEGFR2/FGFR-1 antagonist," Cancer Research, vol. 67, no. 14, pp. 6899-6906, 2007.

[77] J. J. Tentler, E. L. Bradshaw-Pierce, N. J. Serkova et al., "Assessment of the in vivo antitumor effects of ENMD-2076, a novel multitargeted kinase inhibitor, against primary and cell linederived human colorectal cancer xenograft models," Clinical Cancer Research, vol. 16, no. 11, pp. 2989-2998, 2010.

[78] J. Qing, X. Du, Y. Chen et al., "Antibody-based targeting of FGFR3 in bladder carcinoma and $\mathrm{t}(4 ; 14)$-positive multiple myeloma in mice," Journal of Clinical Investigation, vol. 119, no. 5, pp. 1216-1229, 2009.

[79] J. L. Martínez-Torrecuadrada, L. H. Cheung, P. López-Serra et al., "Antitumor activity of fibroblast growth factor receptor 3-specific immunotoxins in a xenograft mouse model of bladder carcinoma is mediated by apoptosis," Molecular Cancer Therapeutics, vol. 7, no. 4, pp. 862-873, 2008. 


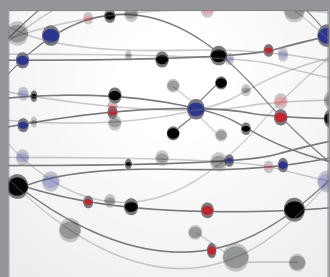

The Scientific World Journal
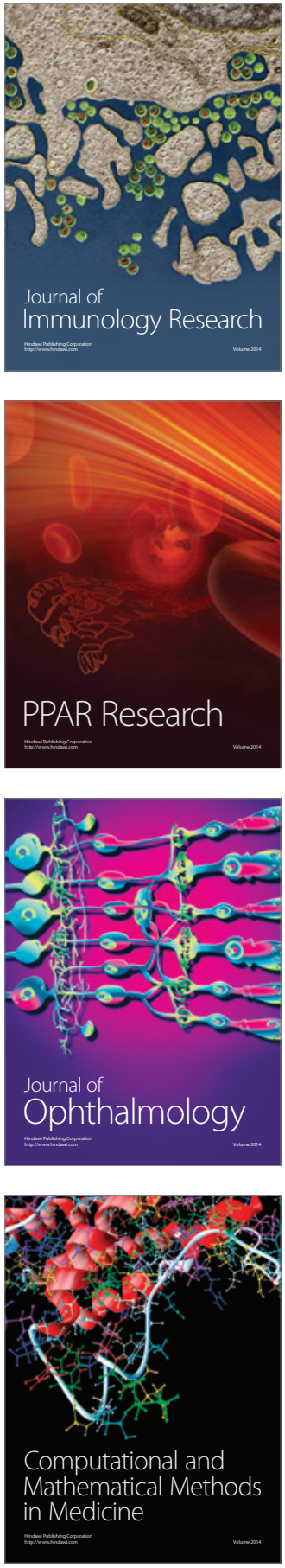

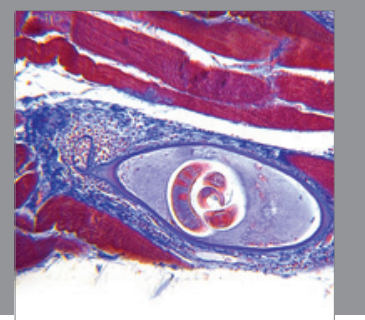

Gastroenterology

Research and Practice
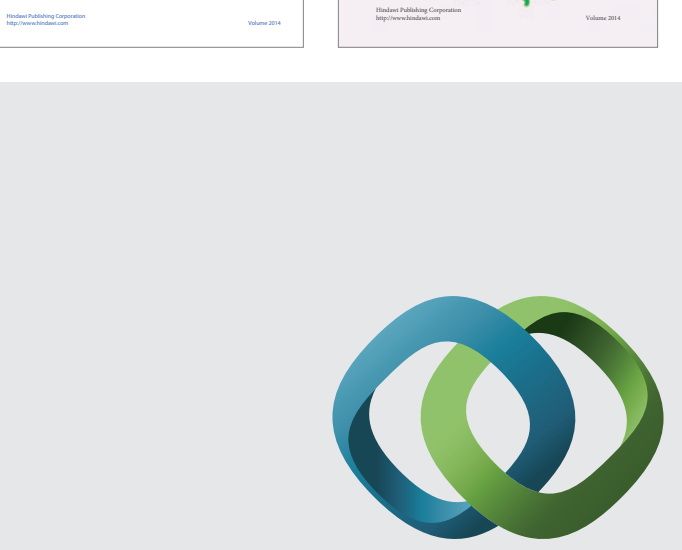

\section{Hindawi}

Submit your manuscripts at

http://www.hindawi.com
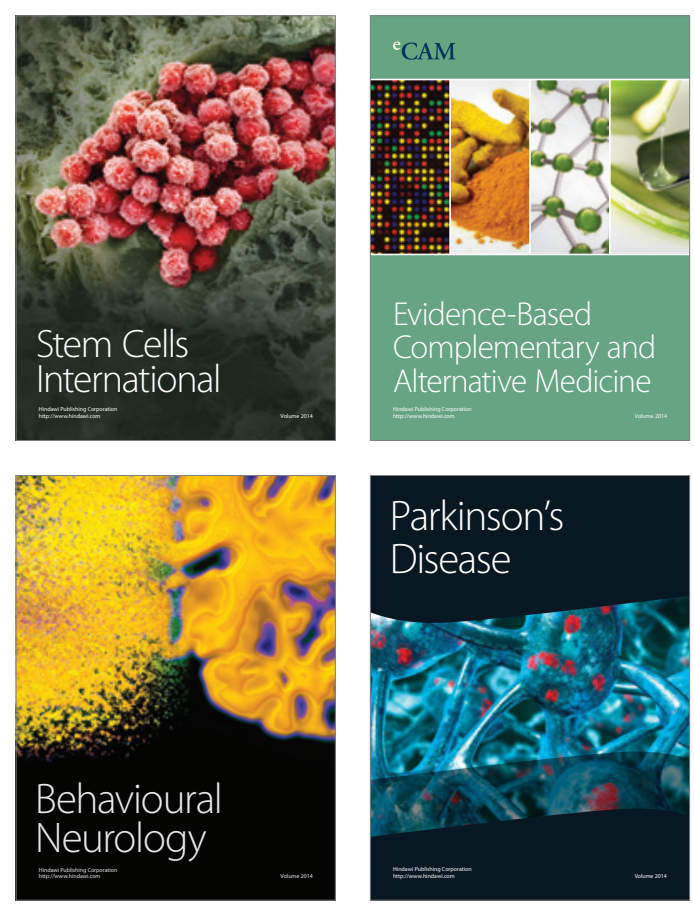

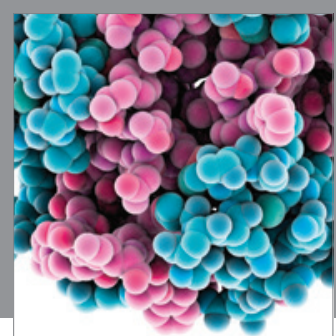

Journal of
Diabetes Research

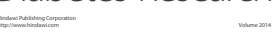

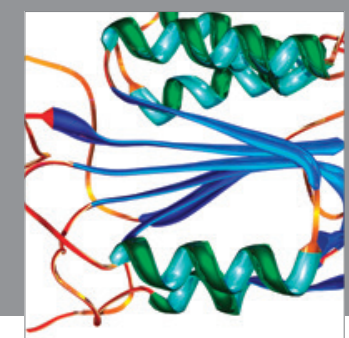

Disease Markers
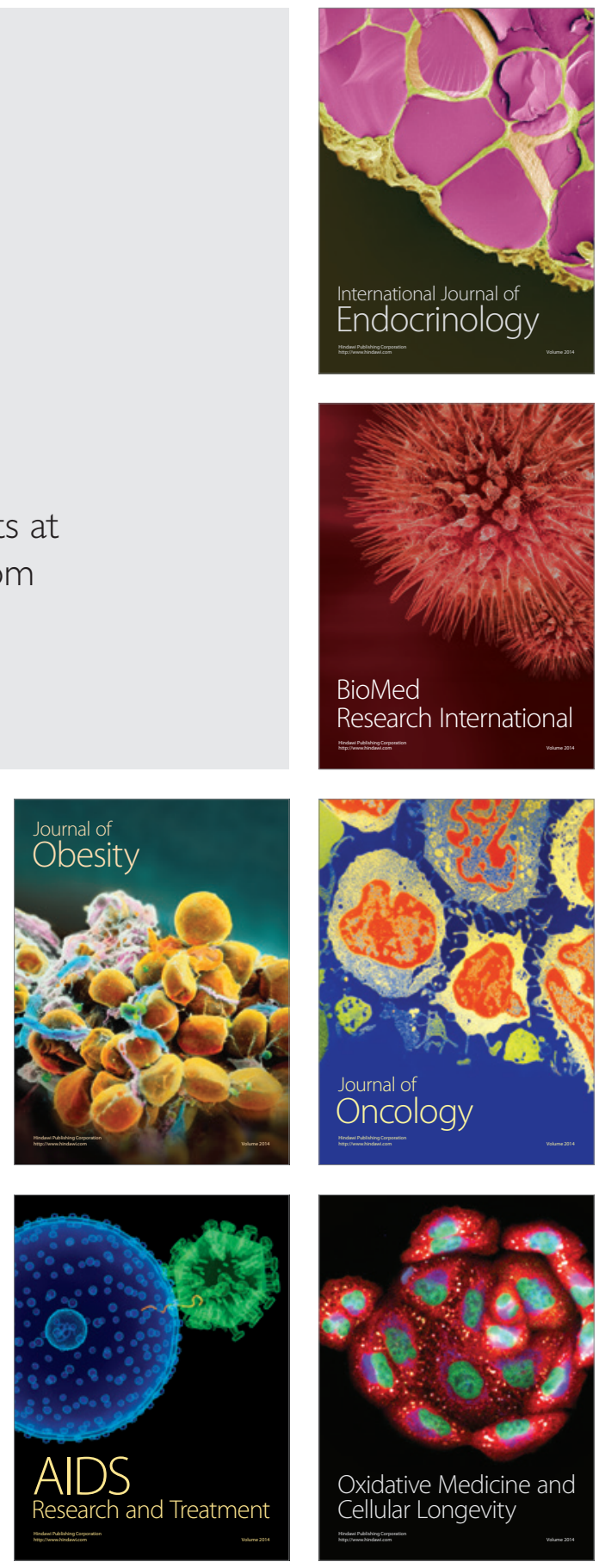